\title{
Intramedullary cement osteosynthesis (IMCO): A pilot study in sheep
}

\author{
Alireza Mirzasadeghi ${ }^{a,}{ }^{*}$, Sri Subanesh Narayanan ${ }^{\mathrm{b}}$, Min Hwei $\mathrm{Ng}^{\mathrm{c}}$, Reza Sanaei ${ }^{\mathrm{d}}$, Chen Hui \\ Cheng $^{\mathrm{d}}$, Mohd Yazid Bajuri ${ }^{\mathrm{b}}$ and Mohammad Hassan Shukur ${ }^{\mathrm{b}}$ \\ ${ }^{a}$ Department of Orthopedics, School of Medicine, Taylor's University, Level 9D, Hospital Sungai \\ Buloh, 47000 Sungai Buloh, Selangor, Malaysia \\ ${ }^{b}$ Department of Orthopedics and Traumatology, University Kebangsaan Malaysia Medical Centre \\ (UKMMC), University Kebangsaan Malaysia (National University of Malaysia), Jalan Ya'acob Latif, \\ Bandar Tun. Razak, 56000 Cheras, Kuala Lumpur, Malaysia \\ ${ }^{c}$ Tissue Engeenering Centre, University Kebangsaan Malaysia Medical Centre (UKMMC), University \\ Kebangsaan Malaysia (National University of Malaysia), Jalan Ya'acob Latif, Bandar Tun. Razak, \\ 56000 Cheras, Kuala Lumpur, Malaysia \\ ${ }^{d}$ Department of Veterinay Clinical Studies, University Putra Malaysia (UPM), 43400 UPM, Serdang, \\ Selangor, Malaysia
}

\begin{abstract}
The application of bone substitutes and cements has a long standing history in augmenting fractures as a complement to routine fracture fixation techniques. Nevertheless, such use is almost always in conjunction with definite means of fracture fixation such as intramedullary pins or bone plates. The idea of using biomaterials as the primary fixation bears the possibility of simultaneous fixation and bone enhancement. Intramedullary recruitment of bone cements is suggested in this study to achieve this goal. However, as the method needs primary testings in animal models before human implementation, and since the degree of ambulation is not predictable in animals, this pilot study only evaluates the outcomes regarding the feasibility and safety of this method in the presence of primary bone fixators. A number of two sheep were used in this study. Tibial transverse osteotomies were performed in both animals followed by external skeletal fixation. The medullary canals, which have already been prepared by removing the marrow through proximal and distal drill holes, were then injected with calcium phosphate cement (CPC). The outcomes were evaluated postoperatively by standard survey radiographs, morphology, histology and biomechanical testings. Healing processes appeared uncomplicated until week four where one bone fracture recurred due to external fixator failure. The results showed $56 \%$ and $48 \%$ cortical thickening, compared to the opposite site, in the fracture site and proximal and distal diaphyses respectively. This bone augmentative effect resulted in $264 \%$ increase in bending strength of the fracture site and $148 \%$ increase of the same value in the adjacent areas of diaphyses. In conclusion, IMCO, using CPC in tibia of sheep, is safe and biocompatible with bone physiology and healing. It possibly can carry the osteopromotive effect of the CPCs to provide a sustained source of bone augmentation throughout the diaphysis. Although the results must be considered preliminary, this method has possible advantages over conventional methods of bone fixation at least in bones with compromised quality (i.e. osteoporosis and bone cysts), where rigid metal implants may jeopardize eggshell cortices.
\end{abstract}

Keywords: Intramedullary cement osteosynthesis (IMCO), calcium phosphate cement (CPC), biomaterial, fracture, impending fracture, osteoporosis, internal fixation

\footnotetext{
*Corresponding author: Alireza Mirzasadeghi, Department of orthopedics, School of medicine, Taylor's University, Level 9D, Hospital Sungai Buloh, 47000 Sungai Buloh, Selangor, Malaysia. Tel.: 60-10-8944001; Fax: 60-3-56295455; E-mail: alireza.mirzasadeghi@taylors.edu.my/alirezamirzasadeghi@yahoo.com.
}

0959-2989/14/\$27.50 @ 2014 - IOS Press and the authors. 


\section{Introduction}

Bone substitutes have been used for decades in the form of bone void fillers and adhesives (cements) or bone grafts in orthopaedic practice and spinal surgeries. In this study Intramedullary Cement Osteosynthesis (IMCO) is suggested as a new modality for treating the fractures or impending fractures of tubular bones. IMCO recruits the medullary canal of the long bones for injection of biomaterials, with certain characteristics, to provide stability and promote osteosynthesis. The rationale behind IMCO lies in the potential ability of the marrow space to provide supports required for stabilizing the bone, by using injectable biomaterials which after setting, function as a load sharing device, much of the same as other intramedullary implants. Moreover, the ability of some of such biomaterials in promoting osteogenesis may pose an advantage under circumstances where an intervention is required to stabilize an impending fracture as in osteoporotic or cystic bones. A variety of available bone cements have well proven their safety and efficacy in augmenting the metaphyseal bones in conjunction with primary fixation methods e.g. screws and plates [1-5]. Previous evaluations have ascribed high compressive resistance to many of these biomaterials that make them suitable for these anatomic sites; nevertheless, such is expected to vary dramatically when the cement is subjected to bending or torsional forces as in diaphyseal regions. To our knowledge, this is the first study that has investigated the effects of calcium phosphate cements (CPC), when they are introduced massively into the medullary canal of the long bones, as will be explained in IMCO. Although similar methods are previously described for the treatment of fractures in birds [6-8], there are substantial biological, morphological and biomechanical differences between avian and human bones.

Crucial role of the medullary canal in bone blood supply has been known for many years [9]. From three main sources of bone circulation (i.e. nutrient, metaphyseal and periosteal arteries) two of them (nutrient and metaphyseal arteries) traverse through the marrow canal, before approaching the target tissues $[9,10]$. Considering this fact, ensuring a preserved marrow function remains the primary concern to be addressed in every orthopedic procedure. Nonetheless, although application of biomaterials is not a new subject in orthopaedic surgery, the applicability suggested in this method that apparently interferes with medullary circulation, is a new concept and thus requires appropriate animal studies before eligibility for human use.

The hypothesis of the current study was that IMCO using the approach described here would yield clinical success in healing of a transverse tibial fracture with no ill effects subsequent to obliteration of the medullary canal and thereby vascular compromise. In fulfillment of the above hypothesis, the fractures were fixed using "type I" external skeletal fixators to eliminate the possible effects of instability and fragment motion on revascularization and healing processes. This study aimed to introduce IMCO as a new option in the treatment of fractures and will discuss its possible advantages and shortcomings in two sheep using a CPC.

\section{Materials and methods}

\subsection{Animals and design}

This study was performed on two sheep considering similarities between the structure of bones in this animal and human osseous tissue [11]. Tibia was selected due to easier surgical approach and postoperative care. Furthermore, tibia has a rather regular shape and spare curvature that makes it more suitable for three-point bending tests [12]. Calcium Phosphate Cement (Norian SRS, Norian 
Corp., Cupertino, CA, USA) was selected to fill up the medullary canals due to its favourable characteristics and safety $[2-5,13,14]$.

Two sheep were acclimated for a period of two and eight weeks respectively before surgeries. All the protocols and methods used in this study have been approved by the institutional animal care and use committee of the National University of Malaysia.

\subsection{Anesthesia and preparation}

Anesthesia was induced using an intravenous injection of a 1:1 mixture of Ketamine $\mathrm{HCl}$ and Diazepam to effect. Anesthetic maintenance was then carried out following tracheal intubation using Isoflurane in a partial rebreathing system through a universal $\mathrm{F}$ circuit. With the affected limb suspended from a stand, the surgical area was aseptically prepared for surgery from the dorsal midline all the way to the hoof box in a circumferential manner. Prophylactic Cefazolin $\mathrm{Na}(22 \mathrm{mg} / \mathrm{kg})$ was initiated at the time of induction and repeated bid for five days. Analgesia was provided by intravenous Tramadol at a dosage of $4 \mathrm{mg} / \mathrm{kg}$ IV q12h for a period of three days starting right before recoveries followed by a course of intramuscular Meloxicam for another three days.

\subsection{Surgical technique}

A small incision was made over the proximal medial tibia and through an appropriate sleeve a unicortical hole was drilled into the medullary canal, as the entry point for cement injection, by use of a $3.2 \mathrm{~mm}$ orthopedic drill bit. Thereafter, a venting hole was similarly made $5 \mathrm{~cm}$ proximal to the distal end of the tibia. Using a craniomedial approach, a $5 \mathrm{~cm}$ longitudinal skin incision was then made over the tibial midshaft and subcutaneous and crural fascia incised on the same line as the skin. Subsequently tibial transverse osteotomy was performed equidistant from the proximal and distal extremities. After displacement of the fracture ends the medullary cavities were evacuated using a large gauge $\mathrm{Ne}-$ laton catheter and thoroughly rinsed by constant vigorous irrigation and suctioning. Grasping each fracture fragment with a bone holding forceps, we reduced and stabilized the fracture with use of an external fixator. For the first sheep a uniplanar (type Ia) external skeletal fixator was recruited; however as this failed in the course of healing, a biplanar (type Ib) construct (Imex Veterinary Inc, Longview, TX, USA) was adopted in the second animal. After fixation, the marrow canal was once again cleaned off debris through the proximal and distal holes. To allow the cement to be delivered, the tip of a Nelaton catheter was trimmed and inserted into the entry hole. After connecting cement applicator to the catheter, injection was started by withdrawing the tube gradually off the canal. While maintaining injection, cement could be seen flowing through the venting hole and fracture site. Nevertheless injection was stopped when cement started to overflow from the entry point. Excess cement was then carefully wiped off from the operation site. This technique ensured sufficient quantities and length of the cement column within the medulla (Figure 1). Total amount of material consumed using the aforementioned method was $4-5 \mathrm{ml}$ per tibia. Surgical site was then cleaned and closed in layers. A modified Robert Jones bandage applied for five days into the postoperative period.

\subsection{Radiography}

Radiographs ware scheduled as a baseline preoperative $\mathrm{x}$-ray as well as survey orthogonal projections which were repeated at fourth and sixth weeks. However, in case of the first sheep, no radiographs were taken at sixth week due to implant failure and subsequent euthanasia by 4 weeks. 


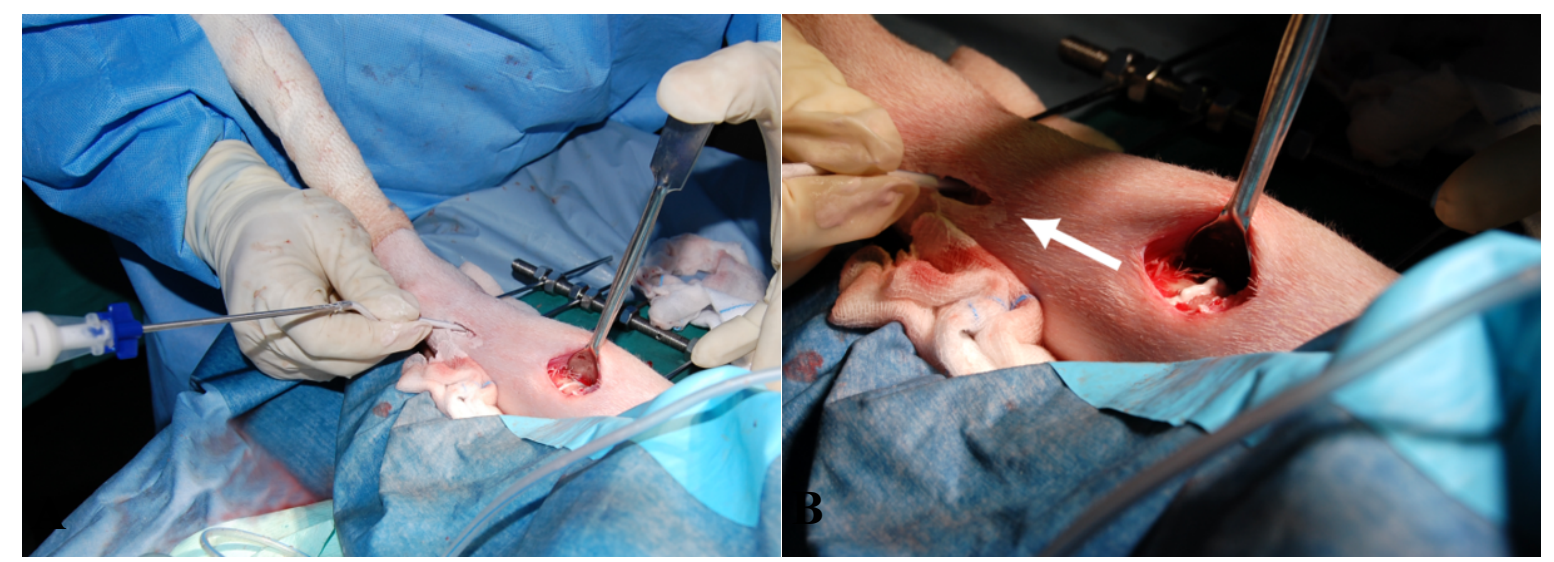

Fig. 1. Photograph from intra-operative procedure, (A) demonstrates cement applicator connected to Nelaton catheter size 14 FG conveyed into whole length of diaphysis; (B) arrow shows direction of catheter withdrawal from far to near segment while injection is continued to ensure enough length of diaphysis is being filled with cement.

\subsection{Biomechanical measurements}

After euthanasia, tibiae were dissected free of all soft tissues, wrapped in a saline-soaked gauze and kept at $4^{\circ} \mathrm{C}$ until use which was within a few hours of euthanasia. Three-point bending strength test was measured using a servohydraulic testing system (Instron 8874, Instron Corp., Canton, MA, USA). The samples were settled horizontally on an adjustable span with flattest surface downward. The two support points were of the rounded type to prevent shearing loads and cutting [15]. Fulcra distance adjusted to $5 \mathrm{~cm}$ from each side of the loading point for all the tests. While applying load at a crosshead velocity of $2 \mathrm{~mm} / \mathrm{min}$ with a $25 \mathrm{KN}$ load transducer until bone fracture, load-displacement graphs were automatically generated. Assuming that bone has a linear elastic behavior, the quantities of ultimate bending strength were measured using prismatic beam bending theory. Biomechanical values of the treated legs with IMCO were compared with normal limbs, as control, for bending strength at the fracture site as well as a point six centimeters proximal to the fracture site. These points were the most distant part of the diaphyses, away from fracture site that allowed us to stabilize the bone on the biomechanical device.

\subsection{Morphological and histological evaluations}

Geometry of the treated bones was compared to that of the contralateral side by measuring the diameter of bones and cortical thicknesses at equal levels using a vernier. For evaluating augmentative effect of the cement in IMCO, values were calculated at the level of fracture site as well as six centimeters proximal to the fracture site. These points were the same spots where fractures were introduced during the biomechanical study.

For microscopic evaluation of the harvested bones, three segments of tibiae from the proximal, distal and fracture sites were sharply excised, fixed in $10 \%$ neutral buffered formalin and decalcified in $10 \%$ EDTA. The specimens were cut into halves longitudinally, and subsequently, transverse and axial samples were obtained and embedded in paraffin. These samples were cut into serial sections each four microns thick, and stained with haematoxylin-eosin as well as Masson's trichrome method. The Masson's trichrome staining was used to differentiate details of the cellular events as well as contrast- 
ing collagen and proteinaceous infiltration following operations. Slides were examined with a standard light transmitting microscope (Olympus BX51TF, Japan) for quality of healing, vascular changes and inflammation as well as osseointegration qualities of the cement. Special care was taken to discern areas of poor blood supply or necrosis.

\section{Results}

The results of this research were categorized as clinical, histological and biomechanical findings.

\subsection{Clinical findings}

Both sheep tolerated the operation satisfactorily until week four where the construct failed, and bone fracture recurred in the first sheep. The fracture recurrence led to fragment displacement and subsequent protrusion through the overlying skin. The second sheep was more docile, and post-operative period was uneventful. Both sheep started to bear weight by day one postoperatively.

By four weeks, the medial frame of the external fixator was removed while the cranial frame was left in place until the time of euthanasia that was at six weeks postoperatively. For both sheep, immediate postoperative radiographs showed appropriate alignment. Radiographs of the second sheep demonstrated preserved alignment and fracture union after four weeks (Figure 2). However, at this time, radiographs of the first sheep showed angulation and displacement due to implant failure.

\subsection{Morphological and histological findings}

Gross evaluation of the specimens revealed normal appearance and architecture of the treated bones at different levels. The fracture site in first sheep showed evidence of focal necrosis possibly as the result of skin break down and bone exposure at this area. In both sheep, there was an obvious increase in the diameter of the diaphyses extending almost throughout the whole length of the shaft (Figure 3).

Based on geometric measurements, IMCO with CPC resulted in $56 \%$ and $48 \%$ increase in cortical thickening in fracture sites and non-fracture sites respectively. The diameter of the shaft showed a $21.3 \%$ and $20.6 \%$ increase for the fracture site and the area away from that in the same manner (Table 1). These increased values were rather uniform throughout the whole length of the diaphyses and were not confined to the level of external fixator pin sites.

Histological review of the specimens did not show any sign of infection at the fracture site or different parts of the bone-cement interface. Osteoclasts could be recognized extensively, and in some sections they appeared to be directly on the surface of the cement in locations suggestive of active mineral resorption (Figure 4A).

Adjacent to the osteoclasts were areas of prompt osteoblastic activity with associated osteoid and new bone formation with woven appearance. There was also extensive evidence of angiogenesis within the new bone and old bone areas. These new vessels were often found to be confined within circumferential lamellae of bone giving the impression of developing haversian systems (Figure 4B). Histologically, this osteon-like architecture resembled the process of normal bone remodeling. This process was evident for the whole length of the bone-cement interface in the diaphyses. In sheep 1, the area of bone exposed to the skin, due to implant failure, showed expected osteonecrosis; nevertheless, there was no sign of infection or osteomyelitis in the surrounding bone. 


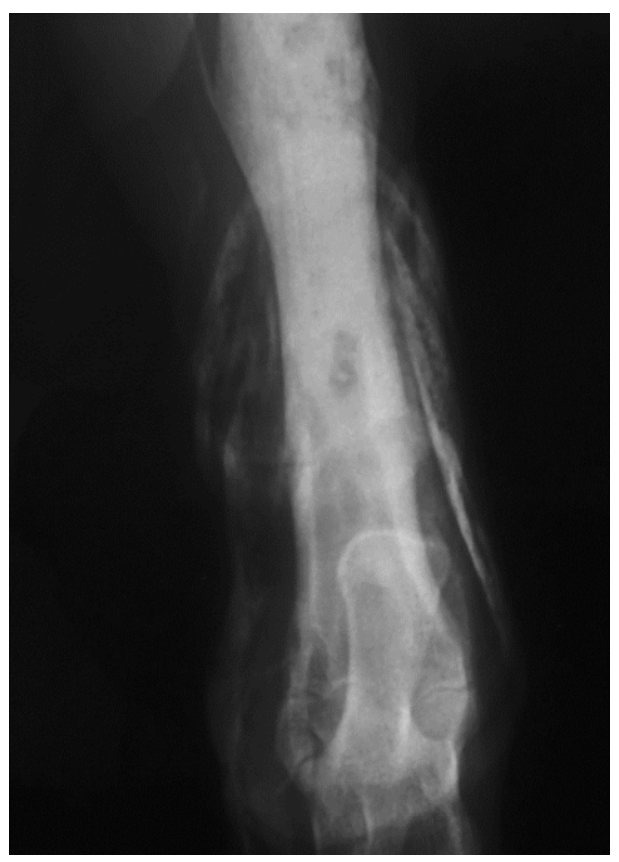

Fig. 2. Anteroposterior (AP) radiograph of the united bone of the sheep 2 at sixth week. Cement incorporated in bone remodeling and some gaps between bone and cement have been filled.

\section{Table 1}

Average diaphyseal and cortical thickness in the areas of fracture site and six centimeters away from the fracture site (values in millimetre)

\begin{tabular}{lllll}
\hline & Diaphysis diameter* & & Cortical thickness** & \\
\cline { 2 - 5 } & Fracture site & Non-fracture site & Fracture site & Non-fracture site \\
\hline \multirow{2}{*}{ IMCO } & 21.3 & 19.3 & 8.0 & 6.1 \\
& $(20.5-22.1)$ & $(16.5-22.1)$ & $(7.4-9.6)$ & $(4.5-7.5)$ \\
\hline \multirow{2}{*}{ Control } & 17.2 & 15.7 & 4.5 & 3.7 \\
& $(16.2-18.2)$ & $(13.2-18.2)$ & $(4.2-5.0)$ & $(3.1-4.0)$ \\
\hline \multirow{2}{*}{ Difference } & 4.1 & 3.6 & 3.5 & 2.4 \\
& $(21.3 \%)$ & $(20.6 \%)$ & $(56 \%)$ & $(48 \%)$ \\
\hline
\end{tabular}

*Diaphyseal diameter is defined as the average of sagittal and coronal distances.

**Cortical thickness represents the mean of cortical thicknesses in anterior, posterior, lateral and medial plans.

Table 2

Values of bending strength of bone treated with IMCO and control tibia (values in $\mathrm{MPa}$ )

\begin{tabular}{lll}
\hline & Diaphysis & \\
\cline { 2 - 3 } & Fracture site & Non-fracture site \\
\hline IMCO & 173.4 & 107.5 \\
\hline Control & 65.6 & 72.2 \\
\hline
\end{tabular}



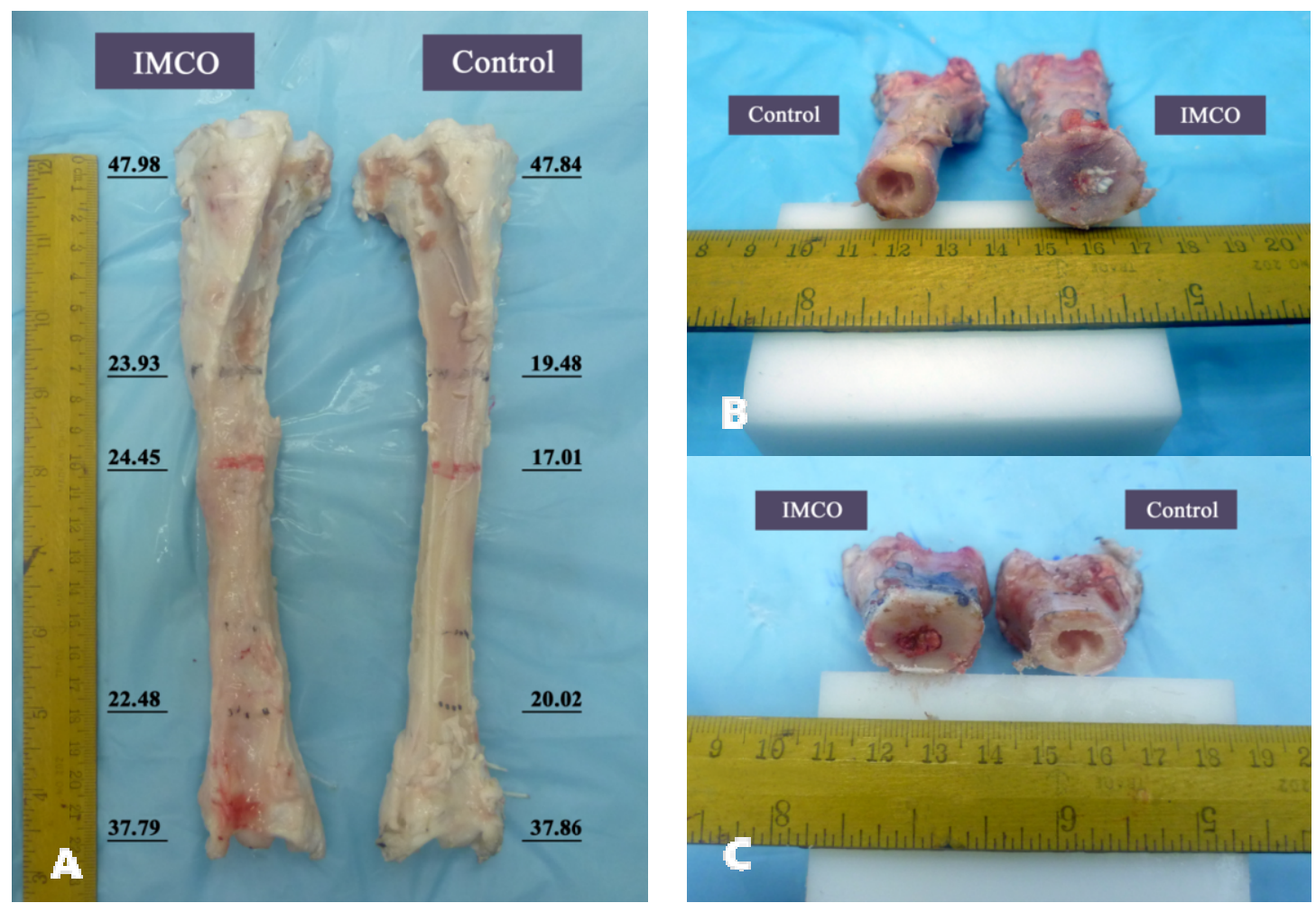

Fig. 3. Morphologic appearance of tibia treated with IMCO compared to contralateral control bone. (A) Numbers are diameter in millimeter at the corresponding levels. Note the treated bone (left side) is thickened in whole length of the diaphysis. Epiphyseal area and length of the bone is unaffected. Transected samples in the right side illustrate how cement is participating in the bone remodeling and resulted cortical mass bulking in the treated bone at the level of fracture site (B) and proximal diaphysis $(\mathrm{C})$.
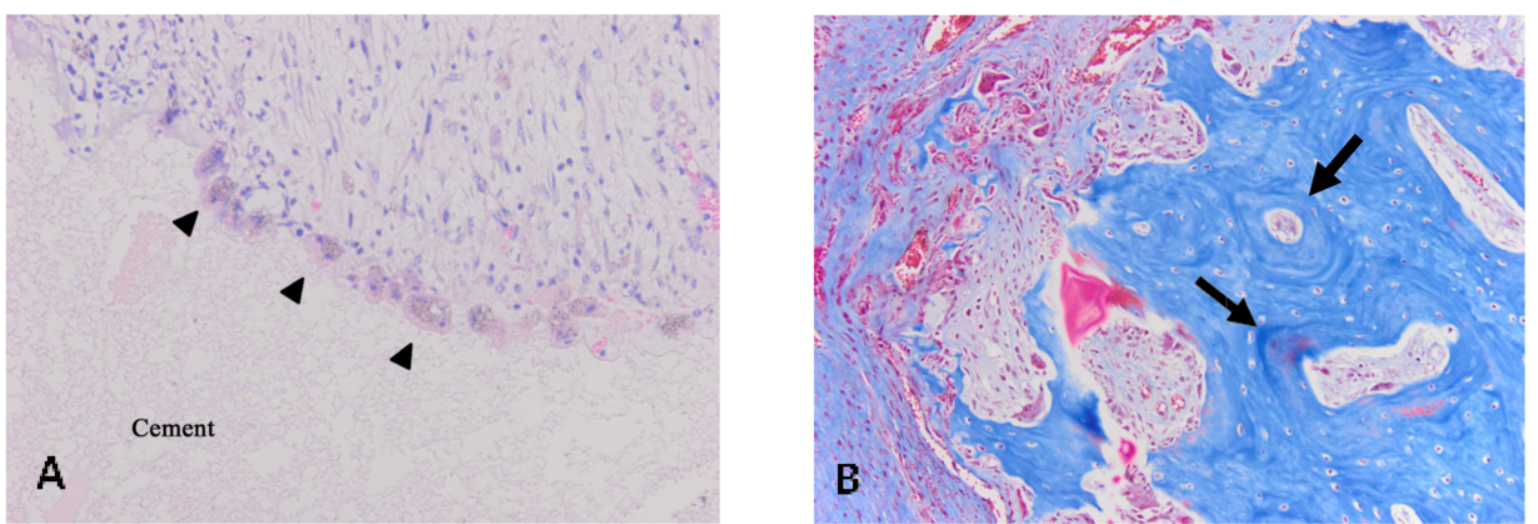

Fig. 4. Photomicrographs of decalcified sections, demonstrating the interface between the cement and the bone. A. Several multinucleated cells (arrow heads) are present invading biomaterial on the surface of the cement. The site and the morphologic feature suggest that they are osteoclasts (haematoxylin-eosin $\times 40$ ). B. Photomicrographs showing later stages of the osteosynthesis at week six post-operatively. The blue stained bone matrix has extensively replaced the CPC. Blood vessels are surrounded by lamellar structures resembling organized haversian system (arrows) penetrating into the cement (Mason trichrome $\times 10$ ). 


\subsection{Biomechanical measurement}

Only tibiae retrieved from sheep 2 were subjected to these evaluations as the fracture recurrence in sheep 2 rendered the specimen unsuitable. Table 2 compares the biomechanical profile of the tibia treated with IMCO and contralateral bone as control. According to these measurements, bone treated with IMCO had $264 \%$ enhancement in bending strength in the fracture site comparing to the normal contralateral bone. This value was $148 \%$ for the area 6 centimeter proximal to the fracture site.

\section{Discussion}

Evaluation of the bone subjected to IMCO for 6 weeks showed $56 \%$ and $48 \%$ cortical thickening in the fracture site and remote areas respectively. This bone augmentative effect resulted in $264 \%$ increase in bending strength of the fracture site and $148 \%$ increase of the same value in the remote areas.

Though the fact that fixation pins might sometimes cause local periosteal reactions, the magnitudes of such bone formation is usually trivial and could not explain these findings. Instead, such observation confirms the osteopromotive effects ascribed to CPCs $[4,13,14]$. Histological investigations, on the other hand, revealed that filling of the intramedullary canals with the solid cement did not disturb normal process of bone revascularization and healing. It could be argued that as there has been no evidence of ischemic osteonecrosis or impairment of healing in this study, obliteration of marrow canal and its conspicuous impact on blood supply should not pose substantial disadvantages. However, proper controls were lacking in this experiment to delineate time differences. Such contradiction could be explained in the context that in response to the disruption of blood supply from the marrow, periosteal vasculature take over and correspond to an extended target area and thereby compensate rapidly for such loss. General pattern of new bone formation showed marked osteoconduction throughout the diaphysis, a feature that is predictable for the CPC that was used in this study. These findings share the same picture of events as previously explained by Elizabeth et al when they used Norian SRS in the metaphysis of the tibia and reconfirm the morphological findings of our study [4].

Based on the findings of this study, IMCO is a safe and biocompatible method with possible advantage of enhancing bone quantity throughout the diaphyseal region. We found this method a technically feasible option that can be performed by following conventional conducts needed for any other orthopaedic procedures. Considering this method as the sole means of fixation with no ancillary complements, and where comparisons should be made between IMCO and routine intramedullary nailing, operation times are expected to be less, as there would be no need for accurate estimation of the entry point or reaming. Furthermore, challenges commonly encountered in advancing of metal implants (reamer or nail) into the narrow medullary canal as well as associated complications such as a stuck nail (or stuck reamer) are not expected. Additionally, the amount of cement used in this model was less than $5 \mathrm{ml}$ per site that is an indication of possible economic advantages. Nevertheless, as potential candidates who may benefit such procedure, patients with osteopenia may require extra costs due to their wider medullary canals.

Degree of load sharing in any device depends on the amount of bone-implant contact $[16,17]$ and IMCO, by filling the canal with biomaterials, provides the maximum bone-cement interface. Hence, theoretically, this method possesses the most load sharing properties among the orthopaedic implants. Increased load sharing properties constitutionally reduces implant failure [17]. Similarly, liquid cements distribute the loads more uniformly; therefore, a reduced risk of stress risers and subsequent complications are predictable. Furthermore IMCO, by using absorbable materials, alleviates the need 
of implant removal and likewise metal-related disadvantages (e.g. some iatrogenic fractures) are less expected. Since the majority of bone substitutes are inherently osteoinductive [1], IMCO may have a distinct benefit in treating fracture or impending fractures in bones severely impaired by osteoporosis or cysts, where introducing metal alloys may further jeopardize thin and eggshell cortices. Cement provides immediate support by replacing the marrow while subsequent participation of the bone substitute in bone remodeling will strengthen the cortices more permanently. This method may also be useful in non-displaced fractures that carry the risk of displacement as well as stress fractures with considerable incidence of non-union and displacement e.g. anterior tibial stress fractures [18].

IMCO has been suggested as a new modality for treating fractures in tubular bone rather than a technique. Hence, judicious evaluation of this method is possible only in the context of several factors including the biomaterial in use, site of fracture and technique chosen. For instance, mechanical strength of each biomaterial, after hardening, determines its capability to provide instant stability. Furthermore surgical techniques, in any given fracture, should be designed accordingly and approved technically.

Seemingly, IMCO is likely able to be used in fractures of non-weight bearing areas such as long bones of upper limb, even though due to the lower shearing resistance, initial short term support and/or delayed load bearing is possibly needed. On the other hand, osteopromotive effects of these biomaterials, that may shorten the period of required support, should be taken into account. However, further biomechanical studies in this regard are needed to warrant clinical success.

Moreover, rapid advances in the production of new bone substitutes are improving the biomechanical strength of these biomaterials. More recent studies investigated new cements that are injectable and biocompatible with excellent compressive, bending and tensile strength that are comparable with cortical bone $[19,20]$ (comparing to the CPCs that have good compressive strength but are weak when placed under bending and/or tensile forces). However, the choice of such biomaterial requires proper investigation before being approved for use in clinical settings [19].

Limitation of implementing the study in an animal model with a weight bearing limb resulted in the distortion of that data pertaining to the biomechanical assessments. This distortion arose from the confounding influence of external fixator, which made the pure biomechanical evaluation of the IMCO impossible. The estimation of instant stability provided by this method needs elimination of this supportive effect and in the best situation can only be performed in human clinical setting. Furthermore, the limited number of samples in this investigation undervalues the statistical significance of the results, a shortcoming that can be a point of improvement for further studies.

\section{Conclusion}

According to the findings of this study, IMCO using CPC in tibia of sheep is safe and biocompatible with bone physiology and healing. It possibly can carry the osteopromotive effect of the CPCs to provide a sustained source of bone augmentation throughout the diaphysis. Bending strength of the treated tibia showed a surprising magnitude of added strength that was almost twice as that of the control value. It emphasizes the potential role of IMCO in treating fractures and impending fractures in bones with compromised quality. However, these results demand further investigation, on more samples and without disturbing effects of the external fixator, to be proved.

Newer bone cements such as biocompatible glasses may fulfill all three requirements of IMCO, i.e. moldable, safe and strong, however, they need further investigated in different in vivo models before widespread clinical applications. Meanwhile, the widespread introduction of bone cements as new os- 
teosynthesis devices is likely to encourage a common interest in producing newer biomaterials with perfect capabilities. In that case this question is raised that whether, in future, IMCO will be evolved to the treatment of choice for many of fractures that are currently indicated for metal fixation.

\section{Acknowledgement}

Special thanks are given to Professor Dr. Sharaf Ibrahim for his continued support throughout the course of this study.

\section{References}

[1] W.R. Moore and S.E. Graves, Bain GI: Synthetic bone graft substitutes, ANZ J. Surg. 71 (2001), 354-361.

[2] F. Theiss, D. Apelt, B. Brand et al., Biocompatibility and resorption of a brushite calcium phosphate cement, BioMaterials 26 (2005), 4383-4394.

[3] D.P. Link, J. van den Dolder, J.J.J.P. van den Beucken et al., Evaluation of the biocompatibility of calcium phosphate cement/PLGA microparticle composites, J. Biomed. Mater. Res. A 87 (2008), 760-769.

[4] P.F. Elizabeth, A.G. Steven, W.B. Thomas et al., Biomechanical and histological evaluation of a calcium phosphate cement, J. Bone Joint Surg. Am. 80 (1998), 1112-1124.

[5] P. Lobenhoffer, T. Gerich, F. Witte et al., Use of an injectable calcium phosphate bone cement in the treatment of tibial plateau fractures: A prospective study of twenty-six cases with twenty-month mean follow-up, J. Orthop. Trauma. 16 (2002), 143-149.

[6] A.B. Kuzma and B. Hunter, A new technique for avian fracture repair using intramedullary polymethylmethacrylate and bone plate fixation, J. Am. Anim. Hosp. Assoc. 27 (1991), 239-248.

[7] P.J. Lind, L.A. Degernes, D.E. Olson et al., Bone cement/polypylene rod orthopedic technique, J. Assoc. Avian. Vet. 3 (1989), 203-205.

[8] D.L. Putney, E.R. Borman and C.L. Lohse, Methylmethacrylate fixation of avian humeral fractures: A radiographic histologic study, J. Am. Anim. Hosp. Assoc. 19 (1983), 773-782.

[9] R.W. Jr. Johnson, A Physiological study of the blood supply of the diaphysis, Clin. Orthop. Rel. Res. 56 (1968), 5-11.

[10] J.A. Buckwalter. Musculoskeletal tissues and the musculoskeletal system. in: Turek's Orthopaedics Principles and Their Application. 6th ed., S.L. Weinstein and J.A. Buckwalter, eds., PA: Lippincott Williams \& Wilkins; Philadelphia, 2005, pp. 28-31

[11] E. Newman, A.S. Turner, J.D. Wark, The potential of sheep for the study of osteopenia: current status and comparison with other animal models, Bone 16 (1995), S277-S284.

[12] K.P. Saffar, N. JamilPour and S.M. Rajaai, How does the bone shaft geometry affect its bending properties, Am. J. Appl. Sci. 6 (2009), 463-470.

[13] M.S. Cohen and K. Whitman, Calcium phosphate bone cement-the Norian skeletal repair system in orthopedic surgery, AORN. J. 65 (1997), 958-962.

[14] D. Simpson and J.F. Keating, Outcome of tibial plateau fractures managed with calcium phosphate cement, Injury 35 (2004), 913-918.

[15] T. Jämsä, P. Jalovaara, Z. Peng et al., Comparison of three-point bending test and peripheral quantitative computed tomography analysis in the evaluation of the strength of mouse femur and tibia, Bone 23 (1998), 155-161.

[16] A. Completo, J.A. Simões, F. Fonseca et al., The influence of different tibial stem designs in load sharing and stability at the cement-bone interface in revision TKA, Knee 15 (2008), 227-232.

[17] E.C. Benzel, Biomechanics of Spine Stabilization: Principles and Clinical Practice, McGraw-Hill, New York, 1995.

[18] K.E. Varner, S.A. Younas, D.M. Lintner et al., Chronic anterior midtibial stress fractures in athletes treated with reamed intramedullary nailing, Am. J. Sports Med. 33 (2005), 1071-1076.

[19] E. Mitzner, P.A.H.M. Pelt, C. Mueller et al., Material properties and in vitro biocompatibility of a newly developed bone cement, Mat. Res. 12 (2009), 447-454.

[20] L. Gerhardt and A.R. Boccaccini, Bioactive glass and glass-ceramic scaffolds for bone tissue engineering, Materials 3 (2010), 3867-3910. 